Leading Article

\title{
The chronic fatigue syndrome: a return to common sense
}

\author{
A.M. Denman \\ Division of Immunological Medicine, Clinical Research Centre and Northwick Park Hospital, \\ Watford Road, Harrow, Middlesex HAl 3UJ, UK.
}

The chronic fatigue syndrome has become one of the past decade's causes célèbres, vying with ecological issues for a place in the public perception of real and imagined threats to society and the individual. Perhaps this interest is a manifestation of the malaise of fin de siècle which in other ages has led to outbursts of anarchy ${ }^{1}$ or increased eschatological preoccupation. Fortunately, the passing of time and the cathartic benefits of publication have restored a more reasoned view of chronic fatigue and its attendant problems. Chronic fatigue first became prominent as one of the features of myalgic encephalomyelitis or the 'ME syndrome' and has subsequently been the subject of intense debate. ${ }^{2-5}$ In truth, fatigue is a symptom with many causes and is thus comparable with dyspnoea or dyspepsia. However, in some patients it is the dominant complaint, thereby defining chronic fatigue as a syndrome sui generis in the minds of some observers.

It is entirely logical that viral infections should have received particular attention in seeking the cause of unexplained fatigue. ${ }^{2,6}$ Many patients with chronic fatigue give a history of a preceding infection, and, in these circumstances, the symptoms which accompany fatigue are often characteristic of viral infections. This suspicion is increased when an epidemic resembling a virus infection in an institute or community is followed by a high incidence of this complaint. In considering specific aetiological possibilities, it is logical to suspect Epstein-Barr virus (EBV) infection since, classically, infectious mononucleosis is followed by prolonged physical and mental tiredness, often associated with the other features of the chronic fatigue syndrome. Indeed, it is salutory to reflect how many sufferers from infectious mononucleosis may in the past have been maligned for their allegedly 'functional' illnesses before appropriate laboratory tests became available.

EBV infection also illustrates the well-known difficulty in ascribing any disease to a ubiquitous

Correspondence: A.M. Denman, F.R.C.P.

Received: 27 November 1989 agent unless the sequence of events is incontrovertible. Virtually all individuals acquire EBV infection, and although it is possible to identify patients with recent primary infections by serological methods, it is not really practicable to use such methods to distinguish individuals with symptoms related to past EBV infection from those in whom this infection is clinically irrelevant. ${ }^{7}$ The immediate and often prolonged mental and physical lethargy accompanying infectious mononucleosis are undoubtedly important problems, not least because those affected are often young people engaged in intellectually demanding occupations. However, chronic fatigue can only be attributed to EBV infection in those individuals in whom there is unequivocal evidence that the chronic phase followed a primary infection by this agent. Conversely, EBV cannot be implicated solely on the basis of high titres of those antibodies which simply reflect previous exposure.

Similar considerations apply to chronic fatigue following enterovirus infections, particularly by echoviruses and Coxsackie B virus. ${ }^{8}$ Prolonged symptoms, including fatigue and myalgia, are a common legacy of infections by these agents, which can be incriminated with reasonable security on clinical grounds when the sequence of events is self-evident. Indeed, in some patients muscle pain and easy fatiguability may be so prominent as to suggest a separate diagnostic category, 'myalgic encephalomyelitis' with a putative enteroviral aetiology (Dowsett et al. - this issue'). However, the clinical criteria proposed for its diagnosis do not clearly distinguish it from other forms of post-viral fatigue and only unequivocal evidence for a specific viral aetiology would make the distinction in convincing fashion. This is also a point of practical importance if a form of the syndrome existed in which active physical rehabilitation were contra-indicated. Unfortunately, when a history of this kind is lacking or long past, it is rarely practicable to confirm the diagnosis by isolating the agent or by serological studies. Antibody titres are only helpful if recent infection by a specific strain of enterovirus can be distinguished from persistent responses to serologically related agents. Even then, it is doubtful whether 
serological evidence alone is sufficient to invoke infection by viruses which are endemic in most communities. Such methods are relevant to epidemiological studies but not to establishing the diagnosis in individual patients. Similarly, the detection of enteroviral RNA sequences in muscle biopsies is an experimental approach which is not yet applicable to clinical practice.

Elucidating the causes of unexplained fatigue would be easier if the pathogenesis of this and related symptoms was better understood. Since similar symptoms are encountered in confirmed viral infections, it is tempting, but illogical, to conclude that these must be responsible even when there is no other evidence to this effect. In reality, two opposing aetiological considerations influence the current debate. On the one hand, the symptoms associated with virus infections largely result from abnormally high blood concentrations of interferons and other cytokines induced by the infection. Since many other disorders provoke similar changes, it would be unwise to assume that 'flu-like symptoms' necessarily follow virus infections, even if the agent cannot be identified. Conversely, one should be wary of the assumption that all viral infections are invariably accompanied by the classical symptoms consequent on efficient immune responses such as interferonaemia. The rapid growth of knowledge concerning the genetic control of immune responses to defined viral antigenic determinants (epitopes) makes it predictable that situations will arise in which the clinical reaction is both blunted and perpetuated.

Once one accepts that chronic fatigue is a symptom with many causes, it is easier to judge the plausibility of the many claims for tests considered relevant to the investigation of patients with this problem. Some of these, usually relating to infection by specific infectious agents, are diagnostic in intention, but it is hard to see how any test, however technically reliable, could be universally applicable in this situation. Others relate to alleged immune defects such as impaired $\mathrm{T}$ lymphocyte responsiveness to phytomitogens, ${ }^{10}$ impaired interferon production ${ }^{11}$ and membrane glycoprotein expression by these cells, ${ }^{12}$ and functional deficiency of natural killer (NK) cells. ${ }^{13}$ However, these assays do not distinguish between defects of primary pathogenetic importance, non-specific suppression secondary to physical ill-health, and changes consequent upon the emotional effects of chronic fatigue. The situation is analogous to the alleged impairment of immune function in patients with depressive illnesses. ${ }^{14}$

Without proper definition of the problem it is equally difficult to debate treatment in any rational manner. Thus it was no more likely that acyclovir, ${ }^{15}$ yeast elimination ${ }^{16}$ or any other therapy would prove a universal cure for chronic fatigue than that a diuretic would help all patients with dyspnoea.

Chronic fatigue still poses many problems. Clinical management demands that known causes of chronic fatigue be excluded. ${ }^{5}$ In particular, depressive disorders are an important cause ${ }^{17}$ and can easily be overlooked, especially given the difficulty in distinguishing between fatigue secondary to depression and depression secondary to fatigue. ${ }^{18}$ Once patients with obvious underlying diseases have been identified, four major categories remain. The first includes patients with an initial illness resembling an acute virus infection in whom fatigue is accompanied by consistent disease features, notably myalgia, headaches, lymphadenopathy and low grade fever. ${ }^{3}$ Patients in the second category have similar chronic symptoms, but they have no history of any obvious precipitating infection. A high incidence of concomitant atopic disorders may prove to be a useful diagnostic point. ${ }^{19}$ The third group comprises patients in whom chronic fatigue, sometimes accompanied by myalgia, is a consistent complaint but who lack the other symptoms suggesting an infective aetiology. Finally, there are patients in whom there is a strong suspicion that fatigue reflects stress or other emotional problems but who will not admit to them. However, there are usually other pointers to this aetiology, such as the long duration of the fatigue with no hint of remission and co-existing complaints of great variability. These patients have commonly espoused other popular diagnoses such as 'food allergy' and 'yeast infection'.

Understanding the nature of chronic fatigue depends on elucidating the mechanisms operating in each set of clinical circumstances. Even in patients whose symptoms follow recognized virus infections, more information is needed about the contribution of specific immune responses to different viral epitopes and the immunopathological consequences; currently, it is equally plausible to postulate that chronic symptoms follow selective immunodeficiency in the response to different epitopes or an allergic response to these same antigenic determinants.

In patients in whom there is a strong but unproven suspicion that their symptoms were provoked by virus or other infections, the problem is to identify novel agents or abnormal patterns of response to ubiquitous agents. Isolating a novel virus does not by itself establish that this is the causative agent any more than does the detection of antibodies to known agents, as recent experience with human herpesvirus type 6 testifies. ${ }^{20}$ Progress depends partly on microbiological expertise and partly on more extensive knowledge about the epidemiology of chronic fatigue. ${ }^{21}$ Thus it will be recalled that the association of EBV with infectious mononucleosis could not have been made without extensive serological data in a defined population. 
Equally, treatment depends on identifying the specific cause of chronic fatigue in individual patients and defining the patterns of host reactivity which predispose to chronic symptoms. Such knowledge can at least form the basis for rational trials. Thus the anti-viral drug acyclovir proved ineffective in a controlled trial in patients with longstanding chronic fatigue, ${ }^{15}$ although the efficacy of this drug has been established in patients with acute EBV infections. ${ }^{22}$ Controlled trials at this Centre indicate that this drug is often effective in patients with post-viral fatigue of short duration.

Similar considerations apply to attempts at immunotherapy. While interferonaemia accounts for many of the symptoms of viral infections, a deficient interferon response contributes to the persistence of many viruses. Only painstaking dissection of the immune response to defined agents followed by controlled trials will indicate whether the therapeutic administration of interferon alpha or gamma leads to clinical deterioration or improvement.

Even in the present state of ignorance about

\section{References}

1. Tuchman, B.W. The Proud Tower. Hamish Hamilton, London, 1966.

2. Goldenberg, D.L. Fibromyalgia and other chronic fatigue syndromes: is there evidence for chronic viral disease? Semin Arthritis Rheum 1988, 18: 111-120.

3. Holmes, G.P., Kaplan, J.E., Gantz, N.M. et al. Chronic fatigue syndrome: a working case definition. Ann Intern Med 1988, 108: 387-389.

4. Katz, B.Z. \& Andiman, W.A. Chronic fatigue syndrome. J Pediatr 1988, 113: 944-947.

5. Spracklen, F.H.N. The chronic fatigue syndrome (myalgic encephalomyelitis) - myth or mystery? S Afr Med J 1988, 74: 448-452.

6. Bannister, B.A. Post-infectious disease syndrome. Postgrad Med J 1988, 64: 559-567.

7. Hellinger, W.C., Smith, T.F., Van Scoy, R.E., Spitzer, P.G., Forgacs, P. \& Edson, R.S. Chronic fatigue syndrome and the diagnostic utility of antibody to Epstein-Barr virus early antigen. JAMA 1988, 260: 971-973.

8. Yousef, G.E., Bell, E.J., Mann, G.F. et al. Chronic enterovirus infection in patients with postviral fatigue syndrome. Lancet 1988, i: 146-150.

9. Dowsett, E.G., Ramsay, A.M. \& McCartney, R.A. Myalgic encephalomyelitis - a persistent enteroviral infection? Postgrad Med J 1990, 66: 526-530.

10. Behan, P.O., Behan, W.M.H. \& Bell, E.J. The postviral fatigue syndrome - an analysis of the findings in 50 cases. $J$ Infect 1985, 10: 211-222.

11. Lever, A.M.L., Lewis, D.M., Bannister, B.A., Fry, M. \& Berry, N. Interferon production in postviral fatigue syndrome. Lancet 1988, ii: 101.

12. Subira, M.L., Castilla, A., Civeira, M-P. \& Prieto, J. Deficient display of CD3 on lymphocytes of patients with chronic fatigue syndrome. J Infect Dis 1989, 160: 165-166. more precise therapeutic strategies, there is no reason for therapeutic nihilism. The importance of physical and psychological rehabilitation in this disorder has rightly been stressed..$^{23}$ Indeed, the simple reassurance that doctors and other health workers accept the reality of the symptoms and accompanying disability is immensely valuable. Our experience at this Centre strongly suggests that a graded exercise programme under the supervision of an experienced physiotherapist reduces the period of disability and may require a short, initial period of in-patient treatment. When indicated, such programmes should be accompanied by detailed psychological counselling.

Our understanding of chronic fatigue has reached an important stage. It is essential that patients with this disorder be given the same support as any group of patients with a chronic disease. However, progress will only be achieved if the different categories of chronic fatigue are dissected with scientific objectivity and therapeutic reason.

13. Caligiuri, M., Murray, C., Buchwald, D. et al. Phenotypic and functional deficiency of natural killer cells in patients with chronic fatigue syndrome. $J$ Immunol 1987, 139: 3306-3313.

14. Denman, A.M. Immunity and depression. Br Med J 1986 , 293: 464-465.

15. Straus, S.E., Dale, J.K., Tobi, M. et al. Acyclovir treatment of the chronic fatigue syndrome: lack of efficacy in a placebocontrolled trial. N Engl J Med 1988, 319: 1692-1698.

16. Renfro, L., Feder, H.M. Jr, Lane, T.J., Manbu, P. \& Matthews, D.A. Yeast connection among 100 patients with chronic fatigue. Am J Med 1989, 86: 165-168.

17. Manu, P., Lane, T.J. \& Matthews, D.A. The frequency of the chronic fatigue syndrome in patients with symptoms of persistent fatigue. Ann Intern Med 1988, 109: 554-556.

18. Komaroff, A.L., Straus, S.E., Gantz, N.M. \& Jones, J.F. The chronic fatigue syndrome. Ann Intern Med 1989, 110: 407-408.

19. Straus, S.E., Dale, J.K., Wright, R. \& Metcalfe, D.D. Allergy and the chronic fatigue syndrome. $J$ Allergy Clin Immunol 1988, 81: 791-795.

20. Dale, J.K., Straus, S.E., Ablashi, D.V. The Inoue-Melnick virus, human herpesvirus type 6 , and the chronic fatigue syndrome. Ann Intern Med 1989, 110: 92-93.

21. Wessely, S. Myalgic encephalomyelitis - a warning: discussion paper. $J R$ Soc Med 1989, 82: 215-217.

22. Andersson, J., Britton, S., Ernberg, I. et al. Effect of acyclovir on infectious mononucleosis: a double-blind, placebocontrolled study. J Infect Dis 1986, 153: 283-290.

23. David, A.S., Wessely, S. \& Pelosi, A.J. Postviral fatigue syndrome: time for a new approach. Br Med J 1988, 296: 696-699. 